\title{
Auf der Suche nach sich selbst
}

\section{Freiwilligenagenturen könnten zum Wohlfahrtsmix beitragen}

\author{
- Holger Backhaus-Maul und Peter Friedrich
}

\footnotetext{
Holger Backhaus-Maul und

Peter Friedrich sind wissenschaftliche Mitarbeiter an der Martin-LutherUniversität Halle-Wittenberg. Internet http://www.philfak3.unihalle.de/paedagogik/rvo
}

Das Zusammenspiel von Hilfesuchenden und Hilfeanbietenden muss zumeist organisiert werden. Die Freiwilligenagenturen können dabei eine förderliche Rolle spielen - doch zu heterogen ist ibr Erscheinungsbild, wie eine neue Studie zeigt.

Moderne Gesellschaften sind Organisationsgesellschaften (vgl. Schimank 2005). Kollektives Handeln findet in Organisationen und durch Organisationen statt. Insofern wird Engagement dann gesellschaftlich bedeutsam, wenn es in organisierter Form - etwa als bürgerschaftliches Engagement - in Erscheinung tritt (vgl. Enquete-Kommission 2002).

Engagement ist dabei auch Ausdruck sozialen Wandels. Im Zuge dessen erleben wir seit Jahrzehnten eine Pluralisierung von Lebensvorstellungen und Lebensstilen, die sich in einer Vielfalt des Engagements niederschlägt - vom Ehrenamt, über das freiwillige bis hin zum bürgerschaftlichen Engagement. Das plurale und heterogene Engagement von Bürgern trifft auf traditionsreiche, in der Vergangenheit bewährte und in der Gegenwart etablierte Institutionen und Organisationen, wie etwa die Freie Wohlfahrtspflege und die kommunale Selbstverwaltung, die sich - entsprechend des für Institutionen und Organisationen typischen Beharrungsvermögens - langsam und allenfalls inkrementalistisch verändern (vgl. Backhaus-Maul/Speck 2005).

Damit rückt die Frage in den Mittelpunkt, wie zwischen dem sich rasch wandelndem Engagement der Bürger einerseits und routinebasierten Institutionen und Organisationen andererseits überhaupt ein fruchtbarer Austausch möglich wird. In modernen Gesellschaften bedarf es eben nicht nur organisierter Handlungsformen, sondern auch dauerhafter Kommunikation und Verständigung, inklusive Konflikt und Auseinandersetzung.

Wenn sich soziale Systeme, wie etwa Wirtschaft, Wissenschaft und Politik, auseinander entwickeln, sind - bevor Kooperationen angesichts grundlegend divergierenden Logiken von Geld, Wahrheit und Macht, überhaupt erst möglich werden - erhebliche und zugleich risikobehaftete Übersetzungsleistungen erforderlich. Moderne Organisationsgesellschaften bedienen sich dazu so genannter intermediärer Organisationen (vgl. Streeck 1986, Angerhausen u. a. 1998, Boeßenecker 2008). In den hier interessierenden Engagementangelegenheiten empfehlen sich - folgt man ihrem Selbstverständnis - Freiwilligenagenturen als ein Prototyp intermediärer Organisationen.

Freiwilligenagenturen können als »junge « Organisation mittlerweile in einigen Großstädten schon auf eine rund dreißigjährige Tradition zurückgreifen, die bis weit in die Milieus und Strukturen neuer sozialer Bewegungen in den 1970- und 1980-Jahren zurück reicht. Dabei war für die Pioniere unter den Freiwilligenagenturen die Auseinandersetzung mit etablierten Organisation und trägen Institutionen ein zentrales Gründungsmotiv. Die Erfahrungen mit der relativen Geschlossenheit auf Seiten von Institutionen und Verbänden, wie etwa der Freien Wohlfahrtspflege, waren mancherorten ein wesentlicher Grund für die Gründung von Freiwilligenagenturen.

Dieses Anliegen kommt im Selbstverständnis von Freiwilligenagenturen treffend zum Ausdruck, wenn sie sich etwa als Vermittlungsagenturen zwischen Bürgern und Organisationen und als gesellschaftliche Entwicklungsagenturen in Engagementangelegenheiten verstehen (vgl. Bundesarbeitsgemeinschaft der Freiwilligenagenturen 2007, Ebert 2003, Jakob/ Janning 2007).

Erst seit Anfang der 2000er-Jahre finden Freiwilligenagenturen zumindest in einem Teilbereich der Sozialforschung punktuell Aufmerksamkeit. Vor diesem Hintergrund wurden in den Jahren 2009 und 2010 - gefördert durch das Bundes- 
ministerium für Familie, Senioren, Frauen und Jugend - im Rahmen einer bundesweiten und lokal vertiefenden Untersuchung grundlegend und umfassend das Selbstverständnis, die Leistungen und die Entwicklung von Freiwilligenagenturen in Deutschland empirisch untersucht (vgl. zum folgenden Backhaus-Maul/Speck 2011，Speck/Backhaus-Maul/Friedrich/ Krohn 2011). Im Folgenden soll anhand ausgewählter empirischer Befunde der Frage nachgegangen werden, inwiefern Freiwilligenagenturen in Engagementangelegenheiten über ein Potenzial als intermediäre Organisation verfügen und ob sie dieses gegebenenfalls auch zur Entfaltung bringen können.

\section{Selbstverständnis und Leistungen}

Ihrem Selbstverständnis als intermediäre Organisationen zufolge agieren Freiwilligenagenturen als »unabhängige lokale In- ihrer Öffentlichkeitsarbeit sowie ihrer eigenen Weiterentwicklung durch neue Leistungsangebote hohe Bedeutung bei. Das Potenzial von Freiwilligenagenturen erschließt sich somit erst in der Gesamtschau aller Funktionen, Aufgaben und Leistungen und beschränkt sich bei weitem nicht auf die Beratung und Vermittlung einzelner Bürger.

\section{Entwicklung}

Nach einer eher zögerlichen Gründungswelle kam es vor allem Ende der 1990er Jahre im Zuge der beginnenden Engagementdebatte zu einer deutlichen Zunahme der Zahl der Einrichtungen. So existierten im Jahr 2001 deutschlandweit bereits rund 190 Freiwilligenagenturen (vgl. Bundesministerium für Familie, Senioren, Frauen und Jugend 2002).

Knapp zehn Jahre (2009) später weist unsere Untersuchung bereits 360 Freiwilligenagenturen aus, was annähernd einer

\section{»Freiwilligenagenturen als >Alleskönner drobt die Profillosigkeit«}

frastruktureinrichtungen « an den »Schnittstellen « zwischen engagementinteressierten Bürgern, gemeinnützigen Organisationen sowie Kommunalpolitik und Kommunalverwaltungen. Durch Informations-, Beratungs-, Vermittlungs- und Qualifizierungsangebote sowie eine breite Palette an Aktivitäten und Maßnahmen in der Öffentlichkeits- und Lobbyarbeit wollen sie eigenen Angaben zu Folge bürgerschaftliches Engagement vor Ort fördern.

Eine der Funktionen von Freiwilligenagenturen besteht in der Information, Beratung und Vermittlung von Bürgern und Organisationen. Im Durchschnitt - so unsere Befunde - wandten sich im Jahr 2008 rund 130 Bürger an eine Freiwilligenagentur, nahmen 72 Bürger eine persönliche Beratung in Anspruch und wurden 46 Bürger an 45 Organisationen vermittelt. Neben der Information, Beratung und Vermittlung von Bürgern und Organisationen messen Freiwilligenagenturen
Verdopplung in knapp einem Jahrzehnt entspricht. Dieses Wachstum von Freiwilligenagenturen erfolgte über die Jahre hinweg allerdings nicht kontinuierlich, sondern war durch erhebliche Schwankungen und vor allem durch Neugründungen und gleichzeitige Schließungen geprägt. Die steigende Zahl an Freiwilligenagenturen im vergangenen Jahrzehnt hat offensichtlich zu einem Wachstum in der Fläche geführt.

\section{Ressourcenausstattung}

Die Untersuchungsergebnisse machen auf eine erhebliche Heterogenität in der finanziellen und personellen Ausstattung sowie eine oftmals unzureichende und mit Risiken behaftete Grundfinanzierung von Freiwilligenagenturen aufmerksam.

So arbeitet fast die Hälfte der Freiwilligenagenturen mit einem Jahresetat von bis zu 10.000 Euro, ein weiteres Drittel mit einem Budget von bis zu 50.000 Euro und weniger als ein Drittel mit einem Jahresbudget von über 50.000 Euro. Dabei dürften die Freiwilligenagenturen mit einem Jahresetat von bis zu 10.000 Euro erhebliche Schwierigkeiten haben, überhaupt eine breite und kontinuierliche Leistungspalette vorhalten und dauerhafte Effekte erzielen zu können.

Ähnlich heterogen und unzureichend ist die personelle Ausstattung von Freiwilligenagenturen: Fast ein Drittel von ihnen arbeitet ohne hauptamtliches Personal, über die Hälfte verfügt immerhin über ein bis zwei hauptamtliche Mitarbeitende und ein vergleichsweise geringer Anteil der Freiwilligenagenturen verfügt über drei und mehr hauptamtliche Mitarbeitende.

\section{Mythen}

Das Verständnis und das Aufgabenprofil der » ambitionierten « unter den untersuchten Freiwilligenagenturen reichen dabei von Selbstbeschreibungen wie »kommunale Entwicklungsagentur « bis hin zur Selbstinthronisierung als »lokale Problemlöser «.

Angesichts dieses weitreichenden und gemessen an den tatsächlichen Möglichkeiten einzelner Freiwilligenagenturen bisweilen überzogenen Selbstverständnisses stellt sich die Frage, wie eine Freiwilligenagentur mit ihrem wenigen Personal, die selbst genannten und zugewiesenen Aufgabenbereiche der Beratung von Bürgern, gemeinnützigen Organisationen und privatgewerblichen Unternehmen, der Initiierung und Entwicklung einer lokalen Engagementinfrastruktur, der engagementpolitischen Lobbyarbeit und nicht zuletzt der Entwicklung engagementbezogener Projekte auch nur ansatzweise erfüllen kann.

Hinzu kommt, dass in den Experteninterviews deutlich geworden ist, dass es in der Regel weder auf Seiten von Kommunen und Ländern noch des Bundes eine institutionalisierte Förderung von Freiwilligenagenturen gibt. Die Förderung seitens der eigentlich für Freiwilligenagenturen im Rahmen der kommunalen Selbstverwaltung zuständigen Kommunen ist - so unsere Fallstudien und Experteninterviews auf Bundesebene - außerhalb großstädtischer Verdichtungsräume in der Regel gering und oftmals nicht auf Dauer angelegt. 
In diesem Zusammenhang verdeutlichen unsere Fallstudien, dass immateriellen Faktoren eine hohe und oftmals unterschätzte Bedeutung für eine erfolgreiche Entwicklung von Freiwilligenagenturen im lokalen Umfeld zukommt. Der erklärte Wille und das bekundete Interesse an einer Freiwilligenagentur von lokalen Akteuren, wie Wohlfahrtsverbänden einerseits sowie von Kommunalverwaltung und Kommunalpolitik andererseits, sind eine wesentliche und geradezu elementare Voraussetzung für eine gelingende Institutionalisierung von Freiwilligenagenturen.

Eine adäquate Ressourcenausstattung sowie eine hinreichende lokale und kommunale Akzeptanz sind aber nicht schon an sich erfolgversprechend, sondern - so die vergleichende Analyse der Fallstudien - bedürfen der Deutung und Ausgestaltung durch das Leitungspersonal und die Mitarbeitenden von Freiwilligenagenturen. Dabei setzen die nach wie vor ausstehende Profilbildung sowie die relativ diffuse und allenfalls ansatzweise erkennbare (Selbst-) Professionalisierung des Personals der Entwicklung von Freiwilligenagenturen deutliche Grenzen. Erschwerend kommt hinzu, dass das unter dem Sammelbegriff »Freiwilligenagenturen « von uns subsumierte Organisationsfeld weder eine einheitliche begriffliche Beschreibung hervorgebracht hat, noch dass die Organisationen in diesem Feld zumindest annähernd vergleichbare Grundstrukturen, Verfahrensweisen und Arbeitsmethoden aufweisen.

\section{Literatur}

Angerhausen, Susanne/Backhaus-Maul, Holger/Offe, Claus/Olk, Thomas/Schiebel, Martina 1998: Überholen ohne einzuholen. Die freie Wohlfahrtspflege in Ostdeutschland, Opladen.

Backhaus-Maul, Holger/Speck, Karsten 2005: Bürgerschaftliches Engagement 2005. Eine empirische Untersuchung des bürgerschaftlichen Engagements in den Mitgliedsorganisationen des Paritätischen Wohlfahrtsverbandes Berlin, Halle/Potsdam.

Backhaus-Maul, Holger/Speck, Karsten 2011: Freiwilligenagenturen in Deutschland. Potenziale auf kommunaler Ebene, in: Nachrichtendienst des Deutschen Vereins für öffentliche und private Fürsorge, 91 Jg., Heft 7 (2011), S. 302-308.

Boeßenecker, Karl-Heinz: Intermediäre Organisationen, in: Bernd Maelicke (Hg.), Lexikon der Sozialwirtschaft, Baden-Baden, S. 520-522.

Bundesarbeitsgemeinschaft der Freiwilligenagenturen (2007): Anleitung zum Aufbau einer Freiwilligenagentur, Berlin.

Bundesministerium für Familie, Senioren, Frauen und Jugend (Hg.) 2002: Freiwilligenagenturen in Deutschland. Ergebnisse einer Erhebung der Bundesarbeitsgemeinschaft der Freiwilligenagenturen (bagfa). Schriftenreihe des Bundesministeriums für Familie, Senioren, Frauen und Jugend. Band 227, Stuttgart.

Ebert, Olaf (2003): Freiwilligenagenturen: Profile, Erfolgskriterien, Probleme. Gutachten für die Enquete-Kommission »Zukunft des Bürgerschaftlichen Engagements « des Deutschen Bundestages, in: Enquete-Kommission »Zukunft des Bürgerschaftlichen Engagements « des Deutschen Bundestages (Hg.), Bürgerschaftliches Engagement in den Kommunen, Opladen. S. 53-108.

Enquete-Kommission »Zukunft des Bürgerschaftlichen Engagements « des Deutschen Bundestages (Hg.) 2002: Bericht. Bürgerschaftliches Engagement: auf dem Weg in eine zukunftsfähige Bürgergesellschaft, Opladen.

Jakob, Gisela/Janning, Heinz (2007): Freiwilligenagenturen als Mittler zwischen Unternehmen und Non-Profit-Organisationen. In: Wirtschaftspsychologie, Heft 1 (2007), S. 14-22.

Schimank, Uwe 2005: Organisationsgesellschaft, in: Wieland Jäger, Uwe Schimank (Hg.), Organisationsgesellschaft. Facetten und Perspektiven, Wiesbaden, S. 19-50. Speck, Karsten/Backhaus-Maul, Holger/Friedrich, Peter/Krohn, Maud 2011: Freiwilligenagenturen und Engagement in Deutschland, Wiesbaden.

Streeck, Wolfgang 1986: Vielfalt und Interdependenz. Überlegungen zur Rolle intermediärer Organisationen in sich ändernden Umwelten, in: Kölner Zeitschrift für Soziologie und Sozialpsychologie, Heft 2 (1986), S. 452-470.

\section{Vergangenheit ohne Zukunft?}

Bemerkenswerterweise gelingt es Freiwilligenagenturen trotz ihres an den Rändern zur Beliebigkeit neigenden Organisationsprofils und ihrer defizitären Ressourcenausstattung sowie der restriktiven institutioneller Rahmenbedingungen zumindest unter günstigen Konstellationen, ihr Potenzial als intermediäre Organisation zur Geltung zu bringen (vgl. Speck/Backhaus-Maul/Friedrich/Krohn 2011). Die flächendeckende Ausdehnung von Freiwilligenagenturen markiert den quantitativen Zwischenstand am definitiven Ende des Aufbauprozesses von Freiwilligenagenturen.

\section{Resümee}

In qualitativer Hinsicht bieten Freiwilligenagenturen nach wie vor ein höchst ambivalentes Bild: So präsentieren sie sich öffentlich als »Alleskönner « mit einem breiten Aufgabenprofil in der Beratung und Vermittlung gegenüber Bürgern, gemeinnützigen Organisationen, Politik und Verwaltung und teilweise sogar privatgewerblichen Unternehmen. Gleichzeitig sind sie in diesem Institutionalisierungsprozess im Profil profilloser geworden. Ihr gesellschafts- und engagementpolitisches Selbstverständnis sind weitgehend verblasst oder abgeschliffen.

Als schlichte Dienstleister in allen Engagementangelegenheiten, die auf jede Nachfrage mit preiswerten Angeboten reagieren, dürfen sie aber wohl keine $\mathrm{Zu}$ kunft als eigenständige und eigensinnige intermediäre Organisationen haben. Aber - so unsere Befunde - niemand auf Seiten von Staat und Kommunen, Politik, und Verwaltung sowie Zivilgesellschaft und Wirtschaft hindert sie daran, ihr Profil weiterzuentwickeln und zu schärfen.

Die vorliegende Untersuchung im Auftrag des Bundesministeriums für Familie, Senioren, Frauen und Jugend ist ein Angebot zur Selbstaufklärung, dass die Beteiligten ignorieren oder goutieren können - sie haben die Wahl. 